\title{
Omega 3 fatty acids supplementation has an ameliorative effect in experimental ulcerative colitis despite increased colonic neutrophil infiltration
}

\author{
Ioannis Varnalidis ${ }^{1}$, Orestis Ioannidis ${ }^{1}$, Elisavet Karamanavi², Zafeiris Ampas ${ }^{3}$, Theofilos Poutahidis ${ }^{2}$, \\ Ioannis Taitzoglou ${ }^{4}$, George Paraskevas ${ }^{5}$ and Dimitrios Botsios ${ }^{1}$
}

${ }^{1}$ Fourth Surgical Department. Medical School. Aristotle University of Thessaloniki. Thessaloniki, Greece. ${ }^{2}$ Laboratory of Pathology. Faculty of Veterinary Medicine. Aristotle University of Thessaloniki. Thessaloniki, Greece. ${ }^{3}$ Department of Rural Development. Democritus University of Thrace. Orestadia, Greece. ${ }^{4}$ Laboratory of Physiology. Faculty of Veterinary Medicine. Aristotle University of Thessaloniki. Thessaloniki, Greece. ${ }^{5}$ Department of Anatomy. Medical School. Aristotle University of Thessaloniki. Thessaloniki, Greece

\begin{abstract}
Purpose: omega 3 polyunsaturated fatty acids have anti-inflammatory properties and can be beneficial in the treatment of inflammatory diseases, such as ulcerative colitis. Dextran sodium sulphate (DSS) colitis in rats appears to mimic nearly all of the morphological characteristics and lesion distributions of ulcerative colitis. The purpose of the current study was to investigate the efficacy of omega 3 fatty acids in the treatment of experimental ulcerative colitis.

Methods: thirty-six Wistar rats were randomly assigned to group A or group B receiving 5\% dextran sulfate sodium (DSS) in their drinking water for eight days. For the next eight days post-DSS, group A animals received tap-water, and group B animals were fed a nutritional solution containing high levels of omega 3 polyunsaturated fatty acids (ProSure ${ }^{\circledR}$, Abbott Laboratories, Zwolle, Netherlands) once per day, administrated with a orogastric feeding tube.

Results: animals fed an omega 3 rich diet exhibited a statistically significant increase in hematocrit and hemoglobin levels, compared to animals drinking tap water, and a trend towards histopathological and clinical improvement, with the administration of omega 3 fatty acids ameliorating epithelial erosion by day 8 post-DSS, but no statistically significant difference was observed between group $A$ and group B animals at 4 or 8 days post-DSS. Also, a statistically significant increase in neutrophil infiltration was observed, as depicted by myelohyperoxidase activity.

Conclusion: our findings support a positive role of omega 3 polyunsaturated fatty acids supplementation in an experimental model of ulcerative colitis despite the increased colonic neutrophil infiltration. Further studies are needed in order to investigate the role of increased neutrophils in colonic mucosa.
\end{abstract}

Key words: Dextran sodium sulphate. Colitis, rat. Nutrition. Fatty acids.

Received: 21-03-2011.

Accepted: 25-05-2011.

Correspondence: Ioannis Varnalidis. Fourth Surgical Department. Medical School. Aristotle University of Thessaloniki. Doiranis 40. 54638 Thessaloniki, Greece.

e-mail: ioanvar@hotmail.com
Varnalidis I, Ioannidis O, Karamanavi E, Ampas Z, Poutahidis T, Taitzoglou I, Paraskevas G, Botsios D. Omega 3 fatty acids supplementation has an ameliorative effect in experimental ulcerative colitis despite increased colonic neutrophil infiltration. Rev Esp Enferm Dig 2011; 103: 511-518.

\section{INTRODUCTION}

Nutritional supplementation with omega $3(\mathrm{~N}-3)$ polyunsaturated fatty acids (N3PUFAs) has been shown to ameliorate the effects of cardiovascular, neurological, autoimmune and inflammatory diseases (1-3). Clinical intervention studies and animal experiments showed that N3PUFAs have anti-inflammatory properties (3), which are based on their ability to antagonize the activity of arachidonic acid, thereby reducing the production of inflammatory and chemotactic derivatives and suppressing cell-mediated immune responses $(3,4)$. Furthermore, N3PUFAs suppress leukocyte activity, cell proliferation, inflammatory cytokine synthesis, natural killer cell activity, antibody synthesis and macrophage surface membrane protein expression (5), and they also act as free radical scavengers (6).

Ulcerative colitis (UC) is a chronic disease characterized by diffuse inflammation of the intestinal mucosa of the large bowel. Multiple hereditary, environmental, microbiological and immunological factors are believed to contribute to the pathogenesis of UC $(7,8)$. Immunoregulatory and pro-inflammatory cytokines appear to play a significant role in UC (9). Immunomodulating agents and corticosteroids have been used to treat UC, although not always with positive outcomes (10). Several lines of evidence indi- 
cated that N3PUFAs could be efficient in the treatment of inflammatory bowel diseases, which would decrease the need for steroids and prevent the recurrence of UC and Crohn's disease (CD) (11). However, there is discordance between these studies which may be related to the effects of other simultaneous treatments, the type and dose of N3PUFA supplements, the mode of patient selection, the type of basic diet and treatment compliance. Therefore, there is an outstanding need to further investigate the role of N3PUFA supplementation in UC and to correlate the clinical course of the disease with macroscopic and microscopic alterations.

An experimental model of UC is induced in rodents by the administration of dextran sulfate sodium (DSS) in the drinking water (12). The DSS-induced colitis causes to the rodents to exhibit many symptoms characteristic of human UC, including bloody feces, diarrhea and weight loss, as well as histopathological lesions characteristic of human $\mathrm{UC}$, including mucosal ulceration and shortening of the large intestine $(12,13)$. DSS-induced UC in Wistar rats has been used widely as an experimental model and appears to mimic nearly all of the morphological characteristics and lesion distributions with human UC. Furthermore, DSSinduced UC in Sprague-Dawley rats shares most clinical, structural and ultrastuctural features with human UC. Thus, the DSS-induced model of UC in rats has proven both valuable and reliable $(12,14)$.

The aims of the current study were to examine the effects of a nutritional solution high in N3PUFAs with regard to the clinical and laboratory course of UC and to correlate these findings with macroscopic and histopathologic features in the Wistar rat model of DSS-induced UC.

\section{MATERIALS AND METHODS}

\section{Animals}

Thirty-six pathogen-free male Wistar rats weighing $250 \mathrm{~g}$ $\pm 50 \mathrm{~g}$ were housed under standard conditions with a controlled 12-h light-dark cycle and free access to food and water. Animal experiments conformed to institutional standards.

\section{Induction of UC model with DSS}

All the rats were randomly assigned to group A or group $\mathrm{B}(n=18)$ rats per group). Both groups received 5\% DSS (molecular weight: $36 \mathrm{kDa} \pm 50 \mathrm{kDa}$; MP Biomedicals Inc., Cleveland, OH, USA) in their drinking water for 8 days until loose stools, diarrhea and macroscopic hematochezia appeared. When the mentioned symptoms appeared the DSS was removed, and 2 rats from each group were sacrificed by cardiac puncture and exsanguinations, which was performed under deep anesthesia by intraperitoneal administration of chloral hydrate. The colonic tissue was examined to confirm the presence of experimental UC.

\section{Intervention}

For the next 8 days post-DSS, group A $(n=16)$ received tap-water, and group B ( $n=16)$ were fed a nutritional solution containing high levels of N3PUFAs -eicosapentaenoic acid (EPA) 0.165 g; ProSure ${ }^{\circledR}$, Abbott Laboratories, Zwolle, Netherlands- once per day administrated with an orogastric feeding tube. Eight rats from each group were sacrificed on the $4^{\text {th }}$ day post-DSS, and the remaining 8 rats from each group were sacrificed on the $8^{\text {th }}$ day post-DSS by cardiac puncture and exsanguination, which were performed under deep anesthesia by intraperitoneal administration of chloral hydrate. The distal colon was examined macroscopically and histopathologically. Blood samples were obtained by cardiac puncture for laboratory tests.

\section{Clinical and laboratory assessment of colonic inflammation}

Animals were observed daily for fluid intake, weight changes and significant symptoms (e.g., loose stools, diarrhea, hematochezia). Body weight and stool consistency were determined daily for all animals. Furthermore, after 8 days of DSS administration and at 4 and 8 days post-DSS, total erythrocyte numbers, hematocrit, hemoglobin concentration, mean corpuscular volume (MCV), mean corpuscular hemoglobin $(\mathrm{MCH})$ and platelets count were determined.

\section{Sample recovery and preparation}

Laparotomy was performed and the colon was visualized and rapidly excised as a whole and placed in a petri dish containing saline solution. The distal colon was photographed and measured. Macroscopic lesions were also determined.

\section{Histology}

Tissues were fixed and embedded in paraffin. Sections were cut at a thickness of $5 \mu \mathrm{m}$ and stained with hematoxylin and eosin (HE). Immunohistochemical staining was performed on $5 \mu \mathrm{m}$ thick, formalin-fixed, paraffinembedded tissue sections. Primary antibodies used for immunohistochemistry included rabbit anti-myeloperoxidase (MPO Ab-1; Thermo Fischer Scientific Lab Vision, Fremont, CA, USA) and rabbit anti-laminin (Laminin Ab1, Thermo Fischer Scientific Lab Vision, Fremont, CA, USA). Antigens were retrieved with Protease XXV for laminin. Heat-induced antigen retrieval was performed with citrate buffer, $\mathrm{pH}$ 6, for myeloperoxidase (MPO). Primary antibody binding was detected with goat antirabbit IgG polymerized horseradish peroxidase (HRP) (Chemicon International, Temecula, CA, USA). Signal 


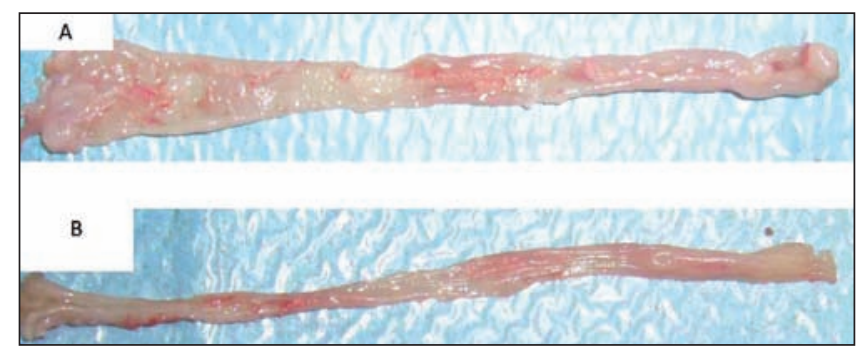

Fig. 1. A. Macroscopic analysis of the distal colon after eight days of drinking water containing $5 \%$ dextran sodium sulphate (DSS) to induce ulcerative colitis (UC). B. Macroscopic appearance of lesions associated with DSS-induced UC in a group B -omega 3 polyunsaturated fatty acid (N3PUFA) diet- animal at 8 days post-DSS.

was detected with diaminobenzidine, and tissues were counterstained with hematoxylin. The colonic lesions were graded on a scale of 0 to 4 with ascending severity (grade 0 : no lesions; grade 1: minimal lesions; grade 2: mild lesions; grade 3: moderate lesions; and grade 4: severe lesions), which was first used by Berg et al. (15), but as it modified later (16).

\section{MPO activity assays}

MPO activity was assayed to determine the extent of neutrophil infiltration into the intestinal mucosa. Neutrophil infiltration was quantitated by assessment of MPOstained slides of intestinal tissue sections. Multiple representative high-power fields (magnification $\times 40$ ) were captured using a Nikon DS-5M-L1 digital camera (Nikon, Tokyo, Japan). Per treatment group random selection of ten images was made. Using the cell count plug-in of the Image J image processing and analysis program $(\mathrm{NIH}$, Bethesda, MD, USA) the identified neutrophils in each image were counted.

\section{Statistical analyses}

For comparisons of numerical data among different groups, a nonparametric ANOVA was performed (KruskalWallis test). All of the above comparisons were performed using Prism Graph Pad programs (GraphPad Software Inc., San Diego CA, USA). All data are expressed as mean \pm standard deviation (SD). Statistical significance was defined as $p<0.05$.

\section{RESULTS}

\section{Macroscopic findings}

Following 8 days of DSS administration, very severe colitis and many hemorrhagic erosions were observed

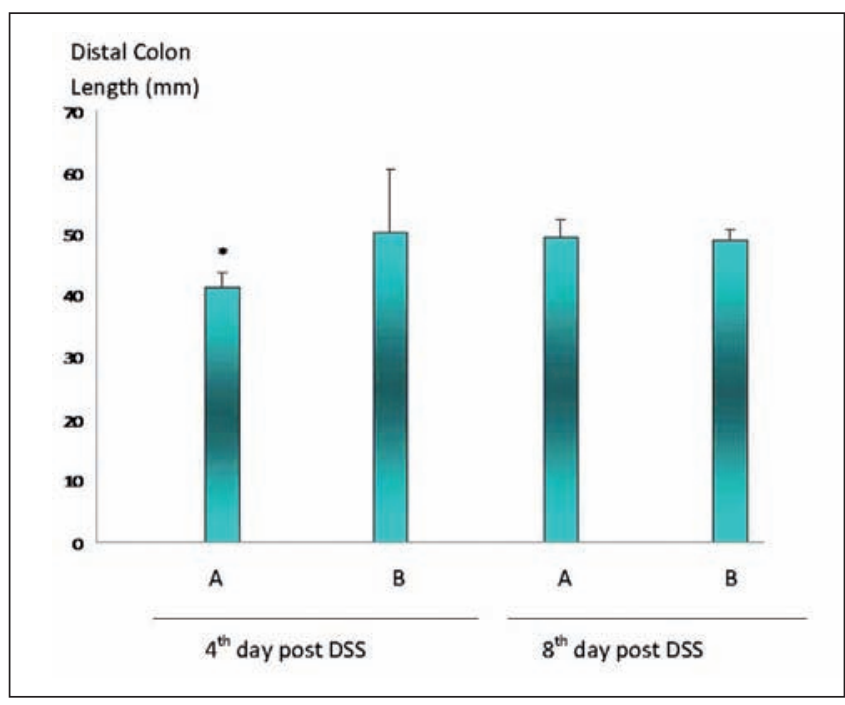

Fig. 2. Mean colon length on day 4 and day 8 post-DSS in group A (tap water) and group $B$ (N3PUFAs diet). ( ${ }^{*} p<0.05$ between the group $A$ in the $4^{\text {th }}$ and $8^{\text {th }}$ day post-DSS).

in the rat colon (Fig. 1A). Notably, administration of a diet rich in N3PUFAs for 8 days post-DSS ameliorated the macroscopic lesions (Fig. 1B). Moreover, the mean colon length in group A (tap water) animals increased significantly from $41.25 \pm 2.5 \mathrm{~mm}$ on day 4 post-DSS to $49.5 \pm 2.8 \mathrm{~mm}$ on day 8 post-DSS $(\mathrm{p}<0.05)$; while in group B (N3PUFA diet) animals, the mean colon length was $50.25 \pm 10.4 \mathrm{~mm}$ and $49 \pm 1.8 \mathrm{~mm}$ on day 4 and day 8 post-DSS, respectively (Fig. 2). After 8 days of treatment, the mean body weights of group A (tap water) and group B (N3PUFA diet) increased slightly from $193.25 \mathrm{~g}$ to $197.75 \mathrm{~g}$ and $199.87 \mathrm{~g}$ to $208.62 \mathrm{~g}$, respectively (Fig. 3).

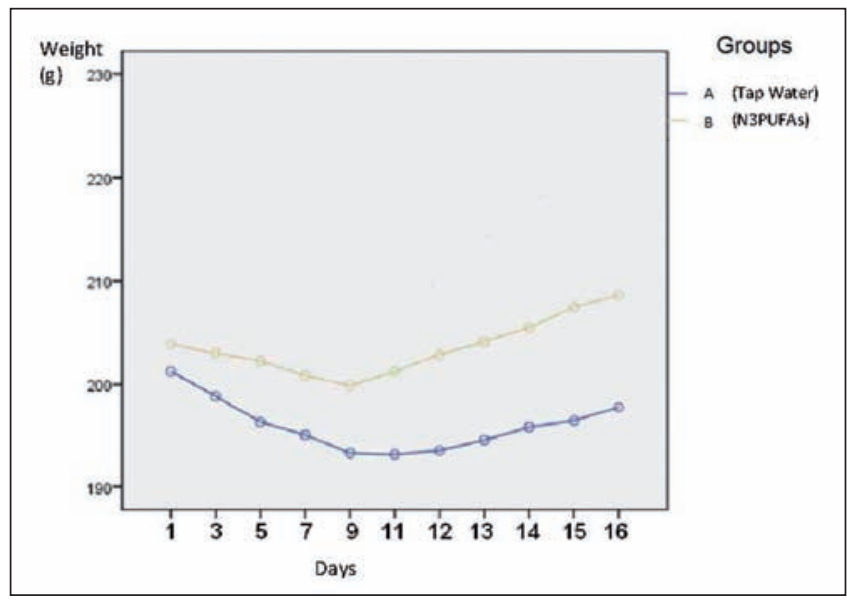

Fig. 3. Mean body weight values in group A (tap water) and group B (omega 3 polyunsaturated fatty acids diet) animals during the 16-day study period. 


\section{Histological findings}

Eight days after the administration of 5\% DSS in both groups, the colonic mucosa appeared edematous, hemorrhagic erosions were scattered along the entire length of the colon and crypt abscesses were focally distributed among damaged glands. Hyperemia, surface blood, ulcers and areas of tissue erosion were observed primarily in the distal colon and rectal mucosa of both groups.

Colonic damage was evaluated in animals which received tap water (group A) or a diet rich in N3PUFAS (group B) for 4 and 8 days after DSS treatment. Typical histopathological findings characteristic of DSS-induced UC included focal erosions, crypt dilatation and heavy inflammatory cell infiltration of the mucosa. 4 days post-DSS, group A (tap water) animals showed focal erosions of the epithelium and slight crypt dilatation with varying degrees of acute inflammatory infiltration, including lymphocytes and polymorphonuclear (PMN) leukocytes of the mucosa (Fig. 4A). Dietary administration of N3PUFAs did not affect the progression of DSSassociated histopathological lesions at four days post-DSS (Fig. 4B). Whereas, group A did not show any significant histopathological improvements at 8 days post-DSS (Fig. $4 \mathrm{C}$ ), in contrast, dietary administration of N3PUFAs ameliorated epithelial erosions at 8 days post-DSS in group B animals (Fig. 4D). Although administration of N3PUFAs ameliorated epithelial erosion by day 8 post-DSS, the colitis injury scores were not statistically significantly $(p=0.190)$ different between group A and group B animals at 4 or 8 days post-DSS (Fig. 5).
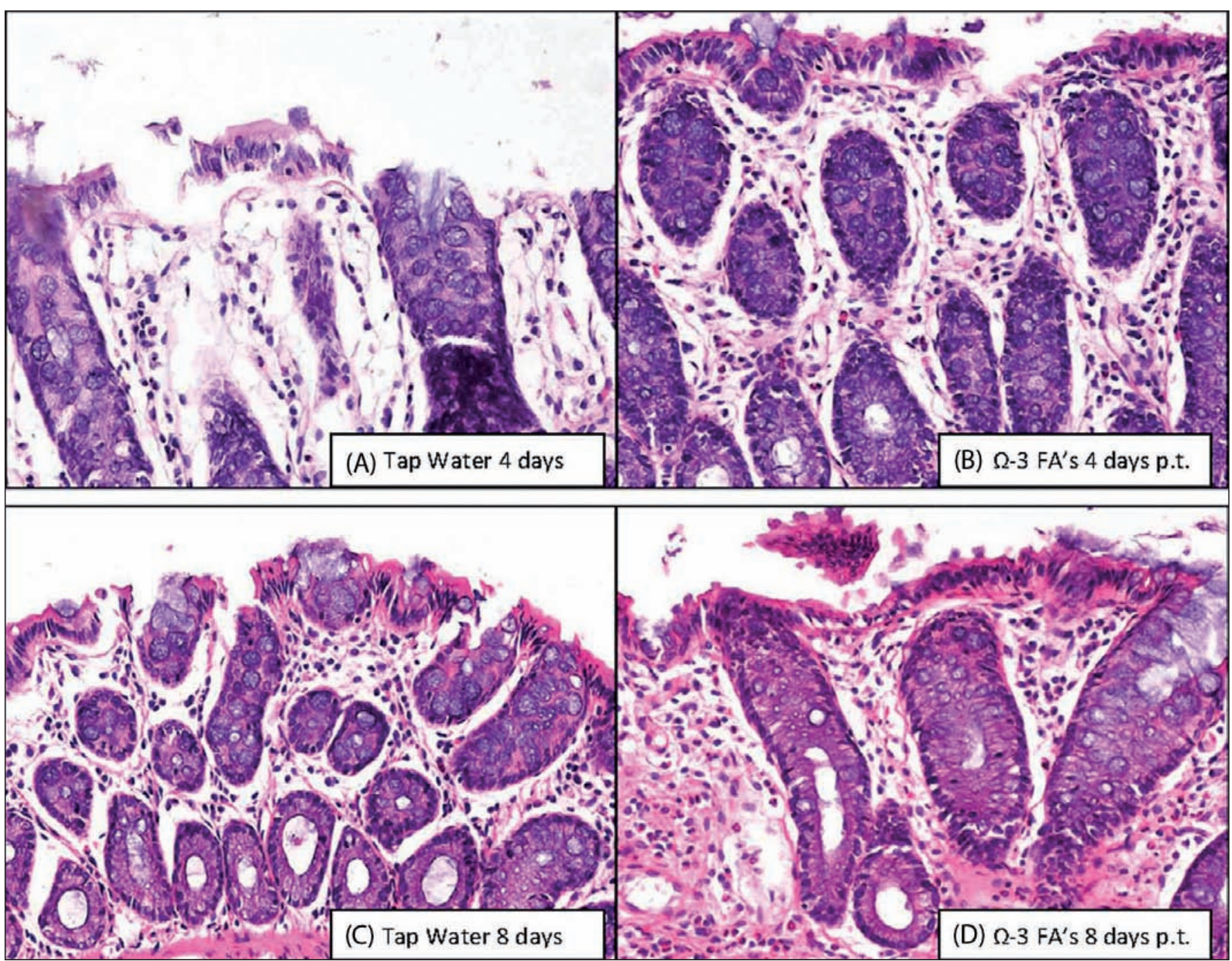

Fig. 4. Hematoxylin and eosin (HE) staining showing characteristic histopathological findings of dextran sodium sulphate (DSS)-induced colitis, which included focal erosions, crypt dilatation and heavy inflammatory cell infiltration of the mucosa in (A) group A (tap water) animals at 4 days post-DSS, (B) group B -omega 3 polyunsaturated fatty acid (N3PUFA)- animals at 4 days post-DSS and (C) group A animals at 8 days post-DSS. (D) Dietary administration of N3PUFAS to group B animals ameliorated epithelial erosions at 8 days post-DSS. 




Fig. 5. Histological grading of dextran sodium sulphate (DSS) - induced ulcerative colitis to quantitate colonic mucosal damage in terms of a colitis score at days 4 and 8 post-DSS in group A (tap water) and group B -omega 3 polyunsaturated fatty acid (N3PUFA) diet-. No statistically significant differences were identified.

\section{MPO activity}

Analysis of MPO-specific immunohistochemical staining of colonic tissue from DSS-treated animals was involved at 4 and 8 days post-DSS. Administration of N3PUFAs was associated with a significant increase in the number of MPO-positive cells in the inflammatory cell infiltrate from DSS-induced colitis in group B (N3PUFA diet) animals at 8 days post-DSS. MPO activity was also increased in the colonic mucosa of group B animals relative to group A (tap water) animals; this increase in MPO activity was statistically significant $(p<0.05)$ (Fig. 6).

\section{Hematological variables}

All of the hematological variables were measured for both groups, including the determination of total leukocyte, ery-

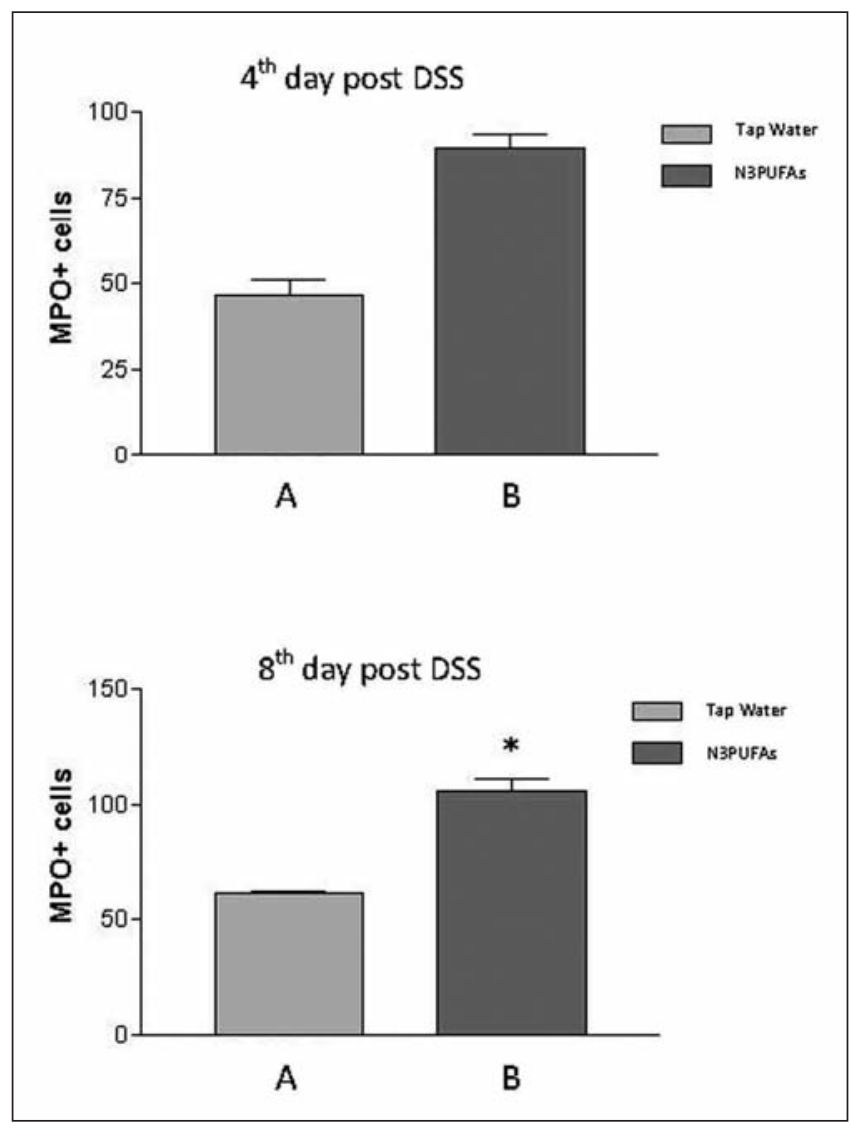

Fig. 6. Effect of $5 \%$ dextran sodium sulphate (DSS) on myeloperoxidase (MPO) activity at 4 days and 8 days post-DSS in group A (tap water) and group B (N3PUFAs diet). ( $\left.{ }^{*} p<0.05\right)$.

throcyte numbers, hematocrit, hemoglobin concentrations, $\mathrm{MCH}$ and MCV after 8 days of DSS administration and at 4 and 8 days post-DSS (Table I). Due to the severity of the hemorrhages associated with DSS administration, the erythrocyte number and hematocrit were diminished in both groups after 8 days of DSS administration (Table I, Fig. 7). Eight days post-DSS, there was a significant increase in the hemoglobin concentration in group B animals $(\mathrm{p}<0.006)$ (Table I, Fig. 7), and there was also a significant increase in platelet (PLT) number $(p<0.002)$ (Table I, Fig. 7). However, $\mathrm{MCV}$ and MCH were not affected, and the MCV was slightly increased at the end of the experimental period.

\section{DISCUSSION}

DSS induced UC in rats is a well-established model of inflammatory bowel disease, which is clinically and histologically similar to human UC $(12,13)$. It is induced by tumor necrosis factor (TNF)- $\alpha$ and IL- $1 \beta$ activation and is characterized by neutrophil mucosal infiltration $(4,9)$. Most rodent models of UC based on DSS administration employ concentrations between 3\% and 7\% DSS in drinking water $(13,17-22)$. 
Table I. Hematological variables at day 4 and day 8 post-dextran sodium sulphate (DSS) in group A (tap water) and group B -omega 3 polyunsaturated fatty acid (N3PUFA) diet- animals relative to control group animals after 8 days of DSS administration (DSS 8 days)

\begin{tabular}{llcrc}
\hline & DSS 8 days & \multicolumn{2}{c}{ Day 4 post-dSS } & \multicolumn{2}{c}{ Day 8 post dSS } \\
& Control & Tap water & N3PUFA & Tap water \\
\hline HCT $(\%)$ & $35.9 \pm 0.4$ & $36.3 \pm 0.3$ & $35.8 \pm 0.9$ & $37.3 \pm 1.1$ \\
HGB $(\mathrm{g} / \mathrm{dL})$ & $12.4 \pm 0.3$ & $12.5 \pm 0.2$ & $12.8 \pm 0.3$ & $13.4 \pm 0.4$ \\
RBC $\left(\times 10^{12} / \mathrm{L}\right)$ & $6.7 \pm 6.2$ & $6.84 \pm 9.1$ & $6.86 \pm 1.1$ & $13.5 \pm 0.3^{*}$ \\
MCV $(\mathrm{fL})$ & $53.2 \pm 0.2$ & $52.5 \pm 0.7$ & $52.5 \pm 0.7$ & $7.99 \pm 2.1$ \\
MCH $(\mathrm{pg})$ & $19.1 \pm 0.2$ & $19.1 \pm 0.3$ & $18.8 \pm 0.2$ & $53.6 \pm 1.1$ \\
PLT $\left(\times 10^{9} / \mathrm{L}\right)$ & $860 \pm 180$ & $870 \pm 160$ & $830 \pm 360$ & $19.2 \pm 0.7$ \\
\hline
\end{tabular}

HCT: hematocrit; HGB: hemoglobin; RBC: red blood cells; MCH: mean corpuscular hemoglobin; MCV: mean corpuscular volume; PLT: platelet count. Data are expressed as mean \pm standard deviation $(S D)\left({ }^{*} p<0.05\right)$.

In our study, after 8 days of 5\% DSS administration in all groups, the colonic mucosa appeared hyperemic, edematous with hemorrhagic erosions and tiny blood coagula. The body weight decreased gradually and the animals exhibited diarrhea and hematochezia.

In our experiment N3PUFAs administration ameliorated epithelial erosion and resulted in a lower colitis injury score, although the difference was not statistically significant. An experimental study (18) has shown that the administration of fish oil, which is rich in EPA and DHA, attenuates colonic inflammation in Wistar rats with DSS-induced ulcerative colitis and significantly improves the colonic microscopic score relative to control rats. However, another study has shown that the administration of N3PUFAs to C57Bl/6 mice with DSS-induced colitis significantly enhanced inflammation, while it also led to a significant decrease in colonic length and body weight (19).

In our study, the observed improvements in clinical manifestations and histopathological scoring associated with N3PUFA administration were accompanied by a significant increase in the number of MPO-positive cells in the inflammatory cell infiltrate of animals with DSS-induced colitis $(p<0.05)$. In a rat model of DSS-induced UC, Lactobacillus fermentum BR11 alleviated the symptoms of colitis, reduced disease activity scores, prevented the shortening of the colon, increased body weight and urine output and prevented crypt hyperplasia, although it was not associated with decreased MPO activity (20). In addition, in a rat model of 2,4,6-trinitrobenzene sulfonic acid (TNBS)-induced colitis, intrarectal administration of mesna resulted in a significant reduction of inflammation and was associated with the persistent attenuation of macroscopic and microscopic changes and a significant increase in inducible nitrogen oxide synthase (iNOS) mRNA expression and MPO activity, suggesting a potential role for iNOS and MPO in the attenuation of colon injury during the chronic phase (23). Furthermore, in another $\mathrm{BALB} / \mathrm{c}$ mouse model (22) of DSS-induced colitis, investigators found that the inflammatory infiltration score peaked at day 7 of DSS administration, the crypt morphology score peaked at day 9 of DSS administration and MPO activity peaked in day 12; all of these changes were statistically significant. Also, DSS administration in C57B1/6 mice led to a significant increase in inflammatory cell infiltration and the crypt morphology score without significantly increasing MPO activity (22). Moreover, in mouse models of both DSS- and TNBS-induced colitis, acute colitis has a lower

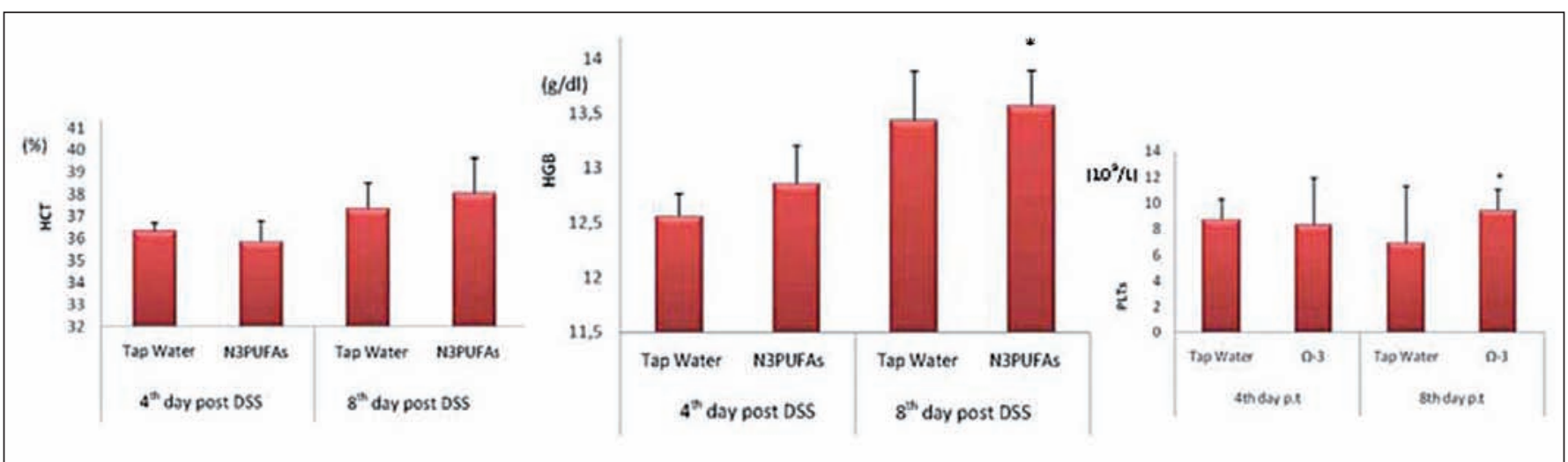

Fig. 7. Hematocrit (HCT) values, hemoglobin (HGB) concentrations and platelet (PLT) numbers at day 4 and day 8 post-DSS. Data are expressed as mean \pm standard deviation (SD) $\left({ }^{*} p<0.05\right)$. 
histological activity index relative to chronic colitis, and MPO activity is increased in acute colitis relative to chronic colitis (23). The results of our study and previous studies have shown that the histopathological scoring of DSSinduced colitis does not necessarily correlate with neutrophil infiltration of the colonic mucosa, which is reflected by MPO activity measurements. A possible explanation for increased neutrophil infiltration includes the increased survival of inflammatory cells due to reduced levels of autooxidative damage (24).

Neutrophils are thought to play a negative role in normal tissue repair as they produce bioactive substances capable of accelerating tissue damage (25), including oxygen radicals (26), digestive enzymes and proinflammatory cytokines (27). However, recent studies have shown that neutrophils may play a beneficial role in wound healing and tissue remodeling and also may have anti-inflammatory properties (28-34). Neutrophils are considered necessary for early steps in wound healing based on the finding that the depletion of neutrophils dramatically delayed wound healing (28). Also, impaired cutaneous wound healing was observed in CXCR-2 deficient mice, which lack PMN leukocyte infiltration (29). Moreover neutrophil recruitment is considered to play an important role in ulcer healing based on the finding that the recruitment of neutrophils to the ulcer site was associated with elevated mucus secretion, thicker regenerative gastric mucosa and increased cell proliferation in an experimental model of acetic acid-induced gastric ulcers (30). Furthermore, neutrophils upregulate VEGF, IL-8, growth-related oncogene (GRO)- $\gamma$ and MCP1 cytokines, which have been reported to stimulate the growth of endothelial cells and fibroblasts (31), and also neutrophils synthesize significant levels of anti-inflammatory mediators, including cytokine inhibitors such as type 2 TNF soluble receptor and IL-1 receptor antagonist in experimental models (32). Moreover, another study demonstrated the production of the IL-1 receptor antagonist, but not the production of pro-inflammatory cytokines TNF $\alpha$, IL-1 $\beta$ and IL-6 (33). Taken together, the results of these studies provide evidence that neutrophils secrete cytokines and chemokines that support the resolution of inflammation and healing of damaged tissue (34) and play a key role in immune regulation by counteracting the dysregulation of the inflammatory process (33), preventing chronic progression and assisting in down-regulating the inflammatory response (28).

In our study, the trend towards improved histopathological scoring combined with a significant increase in neutrophil infiltration, as shown by increased MPO activity, in group $B$ animals fed a N3PUFA-rich diet supports a positive role for neutrophils in the healing and remodeling of damaged tissue. With regard to the haematological variables, both the hematocrit and hemoglobin levels were significantly increased in group B (N3PUFA diet) relative to group A (tap water) at 8 days post-DSS. In support of this finding, a previous study showed that the number of red blood cells, and thus the hematocrit, decreased following seven days of treat- ment with $3 \%$ DSS (35). In our study, we also observed a significant increase in the PLT number for group B (N3PUFA diet) animals relative to group A (tap water) animals at 8 days post-DSS; in contrast, no significant changes in MCV and $\mathrm{MCH}$ levels were observed between groups. With regard to colon length, the current study did not identify any beneficial effects of N3PUFA administration.

Although fish oil administration prior to DSS administration in Wistar rats failed to protect against DSS-induced colitis (36), transgenic mice expressing high endogenous levels of endogenous N3PUFAs were protected against DSS-induced colitis (37), possibly because colon tissue with an increased N3PUFA status generates a higher level of bioactive N3PUFA-derived lipid mediators, such as resolvins and protectins, which may suppress the inflammatory response and enhance mucoprotection (37). Collectively, the results of our study suggest a beneficial role of N3PUFAs in experimental DSS-induced UC that animals fed an N3PUFA-rich diet exhibited a significant increase in hematocrit and hemoglobin levels and a trend towards histopathological and clinical improvement, which was associated with a significant increase in neutrophil infiltration. Our findings support a positive role for neutrophils in healing and remodeling of damaged tissue, which may be the result of the anti-inflammatory effect of N3PUFAs. Circulating N3PUFAs rapidly appear at inflammatory sites that require conversion to resolvins that control excessive neutrophil infiltration, protect organs and foster resolution (38). Furthermore, resolvin E1, an anti-inflammatory lipid mediator derived from EPA, protects against TNBS-induced colitis, increases survival, decreases body weight loss and improves histological scores (39). Therefore, the beneficial effect observed after N3PUFA administration both in human inflammatory bowel disease and in experimental models of UC may be associated with increased levels of resolvin and protectin production.

The present study has certain limitations that need to be acknowledged and addressed when considering the study and its contributions. The small number of subjects per group is a negative factor as it limits the credibility of the statistical analysis. Also the possible variability within the sample may have affected the results. In addition, the fact that certain measurements were depended by the investigator (colon length, histopathological scoring) surely limits the objectivity of the results. So, critically evaluating the results and the whole study is important.

\section{CONCLUSION}

Omega 3 polyunsaturated fatty acids have anti-inflammatory properties and can be beneficial in the treatment of inflammatory diseases, such as ulcerative colitis. Dextran sodium sulphate (DSS) colitis in rats appears to mimic nearly all of the morphological characteristics and lesion distributions of ulcerative colitis. In the current study animals fed an omega 3 rich diet exhibited a statistically significant 
increase in hematocrit and hemoglobin levels, compared to animals drinking tap water, and a trend towards histopathological and clinical improvement, with the administration of omega 3 fatty acids ameliorating epithelial erosion by day 8 post-DSS, but no statistically significant difference was observed between group A and group B animals at 4 or 8 days post-DSS. Also, a statistically significant increase in neutrophil infiltration was observed, as depicted by myelohyperoxidase activity. Our findings support a positive role for neutrophils in healing and remodeling of damaged tissue, which may be the result of the anti-inflammatory effect of omega 3 polyunsaturated fatty acids.

\section{REFERENCES}

1. Kris-Etherton PM, Harris WS, Appel LJ; American Heart Association. Nutrition Committee. Fish consumption, fish oil, omega-3 fatty acids, and cardiovascular disease. Circulation 2002;106:2747-57.

2. Bazan NG. Omega-3 fatty acids, pro-inflammatory signaling and neuroprotection. Curr Opin Clin Nutr Metab Care 2007;10:136-41.

3. Simopoulos AP. Omega-3 fatty acids in inflammation and autoimmune diseases. J Am Coll Nutr 2002;21:495-505.

4. Romano C, Cucchiara S, Barabino A, Annese V, Sferlazzas C. Usefulness of omega-3 fatty acid supplementation in addition to mesalazine in maintaining remission in pediatric Crohn's disease: a double-blind, randomized, placebo-controlled study. World J Gastroenterol 2005; 11:7118-21

5. James MJ, Gibson RA, Cleland LG. Dietary polyunsaturated fatty acids and inflammatory mediator production. Am J Clin Nutr 2000;71(1 Supl.):343S-8S

6. Seidman GE, Bernotti S, Levy E. Nutritional Modulation of Gut Inflammation. In: Labadarios D, Pichard C, editors. Clinical Nutrition: Early intervention. Karger; 2002. p. 41-65.

7. Strober W, Fuss I, Mannon P. The fundamental basis of inflammatory bowel disease. J Clin Invest 2007;117:514-21.

8. Kucharzik t, Maaser C, Lugering A, Kagnoff M, Mayer L, Targan S, et al. Recent understanding of IBD pathogenesis: Implications for future therapies. Basic Science Review 2006;18:1068-83.

9. Sanchez-Munoz F, Dominguez-Lopez A, Yamamoto-Furusho J. Role of cytokines in inflammatory bowel disease. World J Gastroenterol 2008;21:4280-8.

10. Nayar M, Rhodes J. Management of inflammatory bowel disease. Postgrad Med J 2004; 80: 206-13

11. Kozuch P, Hanauer S. Treatment of inflammatory bowel disease: A review of medical therapy. World J Gastroenterol 2008;21:345-77.

12. Erichsen K, Milde AM, Arslan G, Helgeland L, Gudbrandsen OA, Ulvik RJ, et al. Low-dose oral ferrous fumarate aggravated intestinal inflammation in rats with DSS-induced colitis. Inflamm Bowel Dis 2005; 11:744-8.

13. Tahara Y, Ido A, Yamamoto S, Miyata Y, Uto H, Hori T, et al. Hepatocyte growth factor facilitates colonic mucosal repair in experimental ulcerative colitis in rats. J Pharmacol Exp Ther 2003;307:146-51.

14. Gaudio E, Taddei G, Vetuschi A, Sferra R, Frieri G, Ricciardi G, et al. Dextran sulfate sodium (DSS) colitis in rats: clinical, structural, and ultrastructural aspects. Dig Dis Sci 1999;44:1458-75.

15. Berg DJ, Davidson N, Kühn R, Müller W, Menon S, Holland G, et al. Enterocolitis and colon cancer in interleukin-10-deficient mice are associated with aberrant cytokine production and CD4(+) TH1-like responses. J Clin Investig 1996;98:1010-20.

16. Erdman SE, Poutahidis T, Tomczak M, Rogers AB, Cormier K, Plank $\mathrm{B}$, et al. CD4+CD25+ regulatory T lymphocytes inhibit microbiallyinduced colon cancer in Rag2-deficient mice. Am J Pathol 2003; 162:691-702.

17. Kabashima K, Saji T, Murata T, Nagamachi M, Matsuoka T, Segi E, et al. The prostaglandin receptor EP4 suppresses colitis, mucosal damage and CD4 cell activation in the gut. J Clin Invest 2002;109:883-93.

18. Camuesco D, Gálvez J, Nieto A, Comalada M, Rodríguez-Cabezas ME, Concha A, et al. Dietary olive oil supplemented with fish oil, rich in EPA and DHA (n-3) polyunsaturated fatty acids, attenuates colonic inflammation in rats with DSS-induced colitis. J Nutr 2005;135:68794.

19. Matsunaga H, Hokari R, Kurihara C, Okada Y, Takebayashi K, Okudaira K, et al. Omega-3 fatty acids exacerbate DSS-induced colitis through decreased adiponectin in colonic subepithelial myofibroblasts. Inflamm Bowel Dis 2008;14:1348-57.

20. Geier MS, Butler RN, Giffard PM, Howarth GS. Lactobacillus fermentum BR11, a potential new probiotic, alleviates symptoms of colitis induced by dextran sulfate sodium (DSS) in rats. Int J Food Microbiol 2007:114:267-74.

21. Larsson MH, Rapp L, Lindström E. Effect of DSS-induced colitis on visceral sensitivity to colorectal distension in mice. Neurogastroenterol Motil 2006;18:144-52.

22. Alex P, Zachos NC, Nguyen T, Gonzales L, Chen TE, Conklin LS, et al. Distinct cytokine patterns identified from multiplex profiles of murine DSS and TNBS-induced colitis. Inflamm Bowel Dis 2009;15:34152.

23. Shusterman T, Sela S, Cohen H, Kristal B, Sbeit W, Reshef R. Effect of the antioxidant Mesna (2-mercaptoethane sulfonate) on experimental colitis. Dig Dis Sci 2003;48:1177-85.

24. Tsalie E, Kouzi K, Poutahidis T, Abas Z, Sarris K, Iliadis N, et al. Effect of vitamin $\mathrm{E}$ nutritional supplementation on the pathological changes induced in the ileum of rabbits by experimental infection with enteropathogenic Escherichia coli. J Comp Pathol 2006;134:308-19.

25. Dovi JV, Szpaderska AM, DiPietro LA. Neutrophil function in the healing wound: adding insult to injury? Thromb Haemost 2004;92:27580 .

26. Shandall AA, Williams GT, Hallett MB, Young HL. Colonic healing: a role for polymorphonuclear leucocytes and oxygen radical production. Br J Surg 1986;73:225-8.

27. Alzoghaibi MA. Neutrophil expression and infiltration into Crohn's intestine. Saudi J Gastroenterol 2005;11:63-72.

28. Nishio N, Okawa Y, Sakurai H, Isobe K. Neutrophil depletion delays wound repair in aged mice. Age (Dordr) 2008;30:11-9.

29. Schruefer R, Lutze N, Schymeinsky J, Walzog B. Human neutrophils promote angiogenesis by a paracrine feedforward mechanism involving endothelial interleukin-8. Am J Physiol Heart Circ Physiol 2005; 288:H1186-92.

30. Vasconcelos PC, Kushima H, Andreo M, Hiruma-Lima CA, Vilegas W, Takahira RK, et al. Studies of gastric mucosa regeneration and safety promoted by Mouriri pusa treatment in acetic acid ulcer model. J Ethnopharmacol 2008;115:293-301.

31. Theilgaard-Mönch K, Knudsen S, Follin P, Borregaard N. The transcriptional activation program of human neutrophils in skin lesions supports their important role in wound healing. J Immunol 2004;172:768493.

32. Xing L, Remick DG. Neutrophils as firemen, production of anti-inflammatory mediators by neutrophils in a mixed cell environment. Cell Immunol 2004;231:126-32.

33. Schröder AK, von der Ohe M, Kolling U, Altstaedt J, Uciechowski P, Fleischer D, et al. Polymorphonuclear leucocytes selectively produce anti-inflammatory interleukin-1 receptor antagonist and chemokines, but fail to produce pro-inflammatory mediators. Immunology 2006 119:317-27.

34. Borregaard N, Sørensen OE, Theilgaard-Mönch K. Neutrophil granules: a library of innate immunity proteins. Trends Immunol 2007;28:340-5.

35. Kishimoto S, Haruma K, Tari A, Sakurai K, Nakano M, Nakagawa Y. Rebamipide, an antiulcer drug, prevents DSS-induced colitis formation in rats. Dig Dis Sci 2000;45:1608-16.

36. Aslan A, Triadafilopoulos G. Fish oil fatty acid supplementation in active ulcerative colitis: a double-blind, placebo-controlled, crossover study. Am J Gastroenterol 1992;87:432-7.

37. Hudert CA, Weylandt KH, Lu Y, Wang J, Hong S, Dignass A, et al. Transgenic mice rich in endogenous omega-3 fatty acids are protected from colitis. Proc Natl Acad Sci U S A 2006;103:11276-81.

38. Kasuga K, Yang R, Porter TF, Agrawal N, Petasis NA, Irimia D, et al. Rapid appearance of resolvin precursors in inflammatory exudates: novel mechanisms in resolution. J Immunol 2008;181:8677-87.

39. Arita M, Yoshida M, Hong S, Tjonahen E, Glickman JN, Petasis NA, et al. Resolvin E1, an endogenous lipid mediator derived from omega3 eicosapentaenoic acid, protects against 2,4,6-trinitrobenzene sulfonic acid-induced colitis. Proc Natl Acad Sci U S A 2005;102:7671-6. 